\title{
Handwritten Arabic Characters Recognition Using Methods Based on Racah, Gegenbauer, Hahn, Tchebychev and Orthogonal Fourier-Mellin Moments
}

\author{
R. Salouan, S. Safi and B. Bouikhalene \\ Department of Mathematic and Informatic, Polydisciplinary Faculty, \\ Sultan Moulay Slimane University, Beni Mellal, MOROCCO
}

\begin{abstract}
This paper presents for isolated handwritten Arabic characters recognition a comparison between the performances in terms of precision and speediness of six hybrid methods of features extraction based on a combination between profile technique and some efficient moments. More precisely these methods are profile with Racah moment, profile with Gegenbauer moment, profile with Hahn moment, profile with Tchybechev moment and profile with orthogonal Fourier-Mellin moment, finally profile combined in the same time with all these moments. For this purpose we have used for pre-processing the character images the median filter, the thresholding, the centering and the edge detection techniques, while in order to recognize each unknown character we have employed the support vectors machine. The simulation result demonstrates that the most precise method is the profile combined with all moments but in the same time it is the less fast.
\end{abstract}

Keywords: Isolated handwritten Arabic characters, Median filter, Thresholding, Centering, edge detection techniques, Profile method, Racah moment, Gegenbauer moment, Hahn moment, Tchybechev moment, orthogonal Fourier-Mellin moment, Support Vectors Machines

\section{Introduction}

Currently, Optical Character Recognition (OCR) has increasingly attracted the attention of researchers in the field of pattern recognition; in fact in recent years, it is considered undoubtedly an active and dynamic domain in terms of creativity and innovation due to its wide applicability in various areas including postal sorting, bank cheque processing, signatures recognition and automatic data entry, etc.

The fundamental objective of OCR it is to recognize the classes of unknown characters using a stored database of character classes. In practice OCR is fragmented to both principal categories which are printed and handwritten, this last is obviously more difficult than the first, in fact the great variations of writing styles from person to another even so for one given person pose effectively an major challenge front of this type of recognition. In this context, we have interested to isolated handwritten Arabic characters recognition.

Moreover, to achieve a certain character recognition system a succession of operations can be divided into three principal phases. The first one is the pre-processing used for enhancing the image quality, in this sense we have used the median filter, the thresholding, the centering and the edge detection techniques. The second phase is features extraction employed in order to extract some efficient characteristics that each character possesses, we have exploited in this framework six hybrid methods which are the profile combined in first time with Racah moment [1], then with Gegenbauer moment [2] in second time then with Hahn moment [3] in third time then with Tchybechev moment [4] in fourth time then with orthogonal Fourier-Mellin moment [5] in fifth time, 
finally the profile method combined with all these moments in the same time. The last phase is the recognition used to recognize the unknown characters, in order to achieve this phase; we have employed the supports vectors machines. In fact our desired goal in this study is to carry out a comparison between the performances in terms of precision and speediness of all these hybrid methods.

On the other side, several studies has been performed on Latin, Arabic numerals and characters using the profile method [6-9], the moments [10-13], the support vectors machines [14-17].

Furthermore, this paper is organized as follows. In Section 2 the methodology chosen for implementing the desired recognition systems is presented. Section 3 describes how the character images that are preprocessed. Features extraction is explained in Section 4. Recognition phase is given in Sction 5 and experimental results are discussed in Section 6. Finally, in Section 7 the paper is ended by a conclusion.

\section{The Methodology}

The recognition systems that we have used can be presented as follow:

Input numeral
Pre-processing
(Median filter - Thresholding - Centering - Edge detection)
Features extraction
(Profile+Racah - Profile+Gegenbauer - Profile+Hahn - Profile+Tchebychev -
Profile+Orthgonal Fourier-Mellin)
Recognition
(Support vectors machine)
Output numeral

Figure 1. The Proposed Recognition System

\section{Pre-processing}

Pre-processing is the first principal phase of each recognition system that's used for producing a cleaned up version of the quality of the original character image in terms of cancellation of each type of noise and each redundant or undesirable information. In this sens we have opted the following techniques:

- The median filter used for filtering each numeral image.

- The thresholding which serves to render the numeral image contains only the black and white colours according a preset threshold.

- The centering exploited to put the Arabic character justly in center of its image.

- The edge detection that is employed to find the edge of each Arabic character. 
All these techniques will enable as a result to carry out efficiently the features extraction phase.

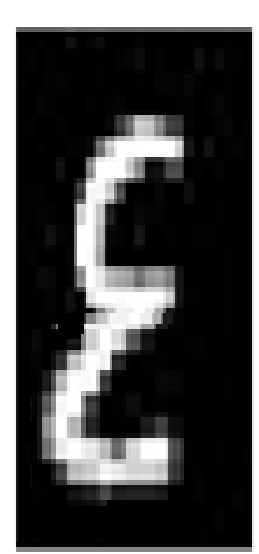

Original image

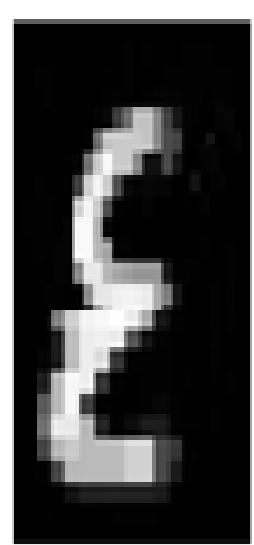

Filtered image

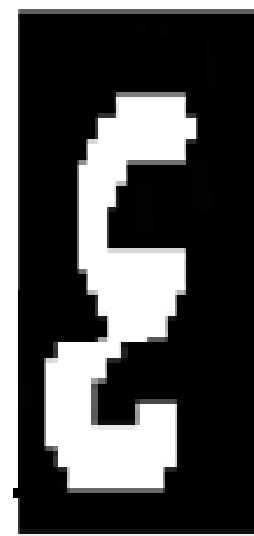

Binarized image

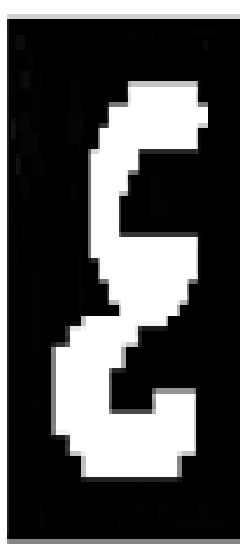

Centered image

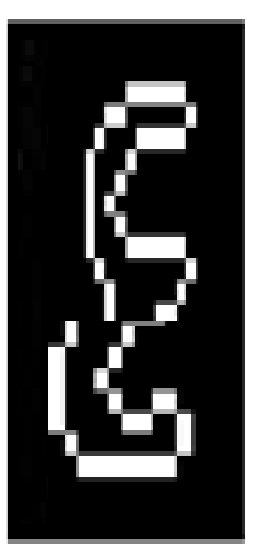

Edge detected of image

\section{Figure 2. Different Techniques used for Pre-Processing the Arabic Character 'Aïn'}

\section{Features Extraction}

Feature extraction is really considered as a one of the major problems of character recognition due to the performance of character recognition system is depends extremely on proper features extracted from character. In pratice an certain features extraction operation is very efficient if it achieved in the same time a strong discrimination interclasses and a weak discrimination intraclasses.

In order to perform this task with success, we have used six efficient hybrid methods based on profile combined with each one of Racah, Gegenbauer, Hahn, Tchybechev, orthogonal Fourier-Mellin moments, afterwards combined in the same time with all these descriptors.

\subsection{Profile Method}

The principle of this method consists to determinate the profile of character in each one of the principal four directions which are right, left, top and low. In order to fix the ideas, first of all given an black image having a size equal to $\mathrm{MxN}$ pixels and containing an character written in white, the mecanism of determination of these profiles articulates on the counting of the number of white pixels in each row situated in left part, in each row localized in right part of character then perform the same procedure but in this time in each column situated in the top part and in each column localized in low part of character; afterwards all these computed numbers of black pixels is saved in a vector having a $2 x(M+N)$ components. Even more, the figure bellow explains the principle of this method: 


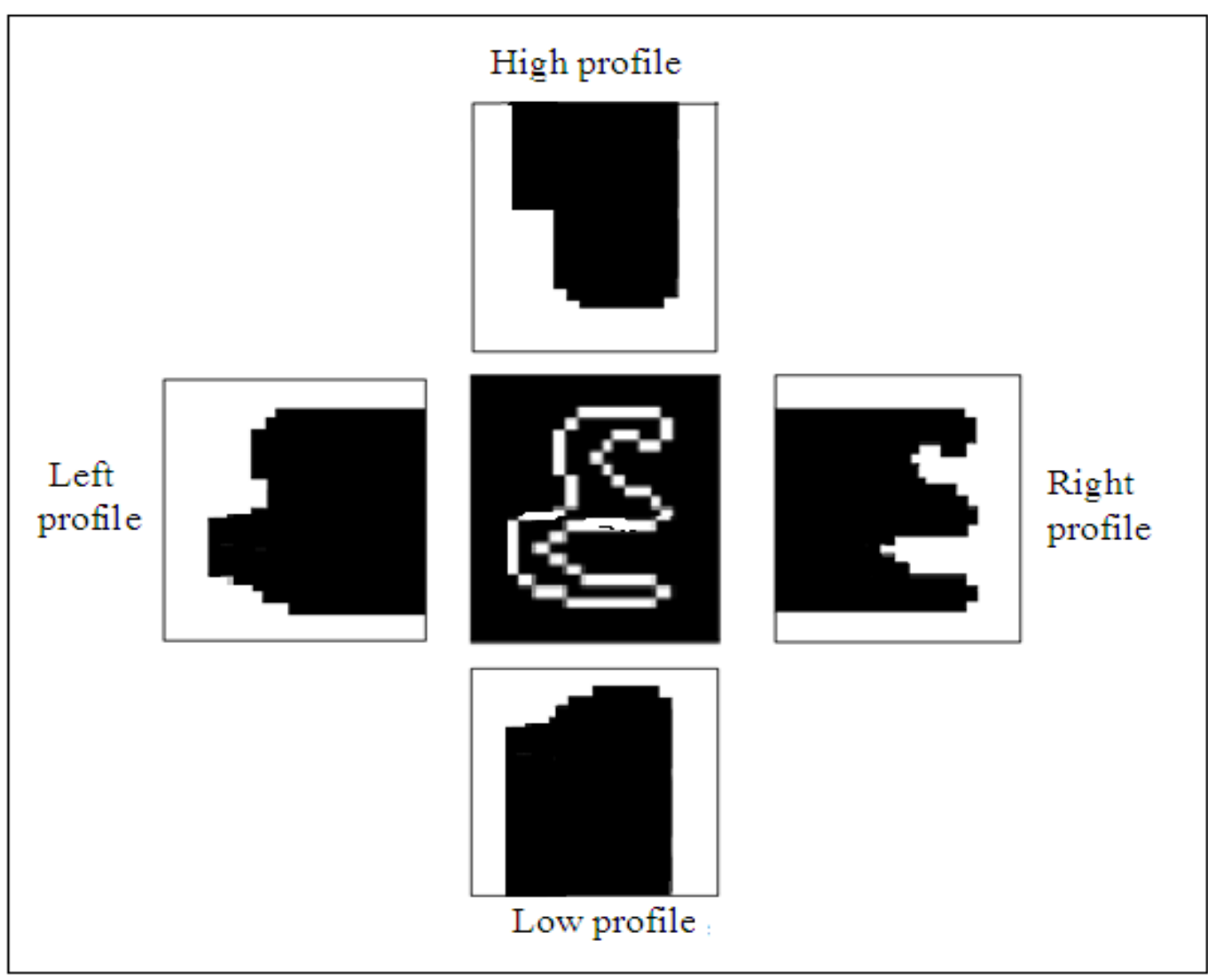

Figure 3. The Principle of Profile Method of Arabic Character 'Aïn'

\subsection{Moments of Images}

Due to their ability to represent statistically global features of an image moments, the moments have found wide applications in the fields of image processing and pattern recognition, in fact the moments are a very powerful mathematical tools used to extract too significant features from patterns.

In this context, we have exploited five powerful moments which are the Racah, Gegenbauer, Hahn, Tchebychev and orthogonal Fourier-Mellin moments.

\subsection{Racah Moment}

The $(n+m)$ order Racah moment of an image $f(x, y)$ with size $N \times N$ pixels is defined as:

$$
\begin{gathered}
U_{n m}=\sum_{x=0}^{a-1} \sum_{y=0}^{b-1} u_{n}^{(\alpha, \beta)}(x, a, b) u_{m}^{(\alpha, \beta)}(y, a, b) f(x, y) \\
n, m=0,1 \ldots L-1
\end{gathered}
$$

The set of weighted Racah polynomial being defined as:

$$
\begin{gathered}
\tilde{u}_{n}^{(\alpha, \beta)}(s, a, b)=u_{n}^{(\alpha, \beta)}(s, a, b) \sqrt{\frac{\rho(s)}{d_{n}^{2}}\left\lfloor\Delta x\left(s-\frac{1}{2}\right)\right\rceil} \\
n=0,1 \ldots \ldots L-1
\end{gathered}
$$

Where: 


$$
\rho(s)=\frac{\Gamma(a+s+1) \Gamma(s-a+\beta+1) \Gamma(b+\alpha-s) \Gamma(b+\alpha+s+1)}{\Gamma(\alpha-\beta+s+1) \Gamma(s-a+1) \Gamma(b-s) \Gamma(b+s+1)}
$$

And:

$$
\begin{gathered}
d_{n}^{2}=\frac{\Gamma(\alpha+n+1) \Gamma(\beta+n+1) \Gamma(b-a+\alpha+\beta+n+1) \Gamma(a+b+\alpha+n+1)}{(\alpha+\beta+2 n+1) ! n !(b-a-n-1) ! \Gamma(\alpha+\beta+n+1) \Gamma(a+b-\beta-n)} \\
n=0,1 \ldots \ldots-1 \\
{ }_{4} F_{3}\left(a_{1}, a_{2}, a_{3}, a_{4} ; b_{1}, b_{2}, b_{3} ; z\right)=\sum_{k=1}^{\infty} \frac{\left(a_{1}\right)_{k}\left(a_{2}\right)_{k}\left(a_{3}\right)_{k}\left(a_{4}\right)_{k}}{\left(b_{1}\right)_{k}\left(b_{2}\right)_{k}\left(b_{3}\right)_{k}} \frac{z^{k}}{k !}
\end{gathered}
$$

And:

$$
\begin{gathered}
u_{n}^{(\alpha, \beta)}(s, a, b)=\frac{1}{n}(a-b+1)_{n}(\beta+1)_{n}(a+b+\alpha+1)_{n} \times_{4} F_{3}(n, \alpha+\beta+n+1, a-s, a+s+1, \beta+1, a+1-b, a+b+\alpha+1 ; 1) \\
\boldsymbol{n}=\mathbf{0}, \mathbf{1} \ldots . . \mathrm{L}-\mathbf{1}, \quad \boldsymbol{s}=\boldsymbol{a}, \boldsymbol{a}+\mathbf{1} \ldots . \boldsymbol{b}-\mathbf{1}
\end{gathered}
$$

And the parameters $a, b, \alpha$, and $\beta$ are restricted to :

$$
-\frac{1}{2} \prec a \prec b, \alpha \succ-1,-1 \prec \beta \prec 2 a+1, b=a+N
$$

And $(a)_{\mathrm{k}}$ is the pochhammer symbol (rising factorial) defined by:

$$
(a)_{k}=a(a+1) \ldots(a+k-1)=\frac{\Gamma(a+k)}{\Gamma(a)}
$$

Where $\Gamma(x)$ is the Gamma function:

$$
\begin{aligned}
& \Gamma(x)=\int_{0}^{\infty} t^{x-1} e^{-t} d t \\
& \forall n \in N, \Gamma(n+1)=n !
\end{aligned}
$$

\subsection{Gegenbauer Moment}

The Gegenbauer polynomials is a class of orthogonal polynomials on the interval [-1, 1] characterized by a single parameter $\lambda$ that allows to change the form of the polynomial.

Hence let $G_{n}(x ; \lambda)$ denote the Gegenbauer polynomial of order $n$ with the parameter $\lambda$, which can be any real number satisfying the restriction of $\lambda>-0.5$.

The Gegenbauer polynomial of order $p$ is defined by:

$$
\begin{aligned}
& (p+1) G_{p+1}(x, \lambda)=2(p+\lambda) x G_{p}(x, \lambda)-(p+2 \lambda-1) G_{p-1}(x, \lambda) \\
& p=1,2 \ldots \ldots, G_{0}(x, \lambda)=1, G_{1}(x, \lambda)=2 \lambda x,-1 \leq x \leq 1, \lambda \succ-0.5
\end{aligned}
$$

Let $f(x, y)$ be an image function, we can define the $(p+q)$ Gegenbauer moment in a two dimensional space as follows: 


$$
A_{p q}=C_{p}(\lambda) \int_{-1-1}^{1} \int_{-1}^{1} f(x, y) G_{p}(x, \lambda) G_{q}(y, \lambda)\left(1-x^{2}\right)^{\lambda-\frac{1}{2}}(1-y)^{\lambda-\frac{1}{2}} d x d y
$$

With the normalizing constant:

$$
C_{p}(\lambda)=\frac{2^{2 \lambda} \Gamma^{2}(\lambda)}{2 \pi} \frac{n !}{\Gamma(n+2 \lambda)}(n+\lambda), \lambda \neq 0
$$

\subsection{Hahn Moment}

The Hahn polynomial of order $\mathrm{n}$ is defined as:

$$
h_{n}^{(\mu, v)}(x, N)=(N+v-1)_{n}(N-1)_{n} \sum_{k=0}^{n}(-1)^{k} \frac{(-n)_{k}(-x)_{k}(2 N+\mu+v-n-1)_{k}}{(N+v-1)_{k}(N-1)_{k}} \frac{1}{k !}
$$

And $(a)_{\mathrm{k}}$ is the pochhammer symbol.

$(\mu, v) \succ(-1,-1)$ are the adjustable parameters For an numerical image $f(x, y)$ with a size $N x N$ pixels the $(n+m)$ the controlling the shape of polynomials. Order of Hahn moment of image is defined as:

$$
\boldsymbol{H}_{m n}=\sum_{x=0}^{N-1} \sum_{y=0}^{N-1} f(x, y) \tilde{h}_{m}^{(\mu, v)}(x, N) \tilde{h}_{n}^{(\mu, v)}(y, N)
$$

$$
m, n=0,1 \ldots . N-1
$$

To avoid numeric fluctuations in moment computation, the Hahn polynomial is scaled by the square norm and the weighting function:

$$
\begin{gathered}
\tilde{h}_{n}^{(\mu, v)}(x, N)=h_{n}^{(\mu, v)}(x, N) \sqrt{\frac{\rho(x)}{d_{n}^{2}}} \\
n=0,1 \ldots . N-1
\end{gathered}
$$

$\rho(x)$ is the weighting function which is given by :

$$
\rho(x)=\frac{1}{\Gamma(x+1) \Gamma(x+\mu+1) \Gamma(N+v-x) \Gamma(N-n-x)}
$$

And the square norrm has the following expression :

$$
\begin{aligned}
& d_{n}^{2}=\frac{\Gamma(2 N+\mu+v-n)}{(2 N+\mu+v-2 n-1) \Gamma(N+\mu+v-n)} \\
& \frac{1}{\Gamma(N+\mu-n) \Gamma(N+v-n) \Gamma(N+1) \Gamma(N-n)}
\end{aligned}
$$

\subsection{Tchebychev Moment}

The Tchebychev moment of order $(p+q)$ of an image function $f(x, y)$ is defined by: 


$$
\begin{gathered}
T_{p q}=\frac{1}{\rho(p, N) \rho(q, N)} \sum_{x=0}^{N-1} \sum_{y=0}^{N-1} t_{p}(x) t_{q}(y) f(x, y) \\
p, q=0,1 \ldots N-1
\end{gathered}
$$

Where ${ }^{t}{ }_{p}(x)$ is the scaled Tchebychev polynomial and satisfies to the following recurrence formula:

$$
\begin{aligned}
& (p+1) t_{p+1}(x)-(2 p+1)(2 x-N+1) t_{p}(x)+p\left(1-\frac{p^{2}}{N^{2}}\right) t_{p-1}=0 \\
& p=0,1 \ldots N-1, x=0,1 \ldots N-1
\end{aligned}
$$

With the initial conditions:

$$
t_{\mathrm{o}}(x)=1, t_{1}(x)=\frac{(2 x-N+1)}{N}
$$

And the squared norrm is given by:

$$
\begin{aligned}
& \rho(p, N)=\frac{N\left(-\frac{1^{2}}{N^{2}}\right)\left(1-\frac{2^{2}}{N^{2}}\right) \ldots\left(1-\frac{p^{2}}{N^{2}}\right)}{2 p+1} \\
& p=0,1 \ldots N-1
\end{aligned}
$$

\subsection{Orthogonal Fourier-Mellin Moment}

The orthogonal Fourier-Mellin moment of order $(n+m)$ of an image $f(x, y)$ is defined in a polar coordinate system over the unit circle as :

$$
\Phi_{n m}=\frac{1}{2 \pi \alpha_{n}} \int_{0}^{2 \pi} \int_{0}^{1} f(r, \theta) Q_{n}(r) e^{-j m \theta} r d r d \theta
$$

Where $m=\ldots . .-2,-1,0,1,2,3 \ldots$. is a circuler harmonic order and is a radial polynomial in $r$ and degree $n$ and defined by :

$$
Q_{n}(r)=\sum_{s=0}^{n} \alpha_{n s} r^{s}
$$

Or :

$$
Q_{n}(r)=(-1)^{s+n} \frac{(n+s+1) !}{(n-s) ! s !(s+1) !}
$$

Therefore, the normalization constant in (24) is:

$$
\alpha_{n}=\frac{1}{2(n+1)}
$$




\section{Recognition}

The SVM aim [18] is to determine a classifier or decision function that separates between both different classes and maximizes the distance between them knowing that the first class contains a set of vectors having a label equal to 1 and the second class includes the other vectors that is labeled by the value -1 .

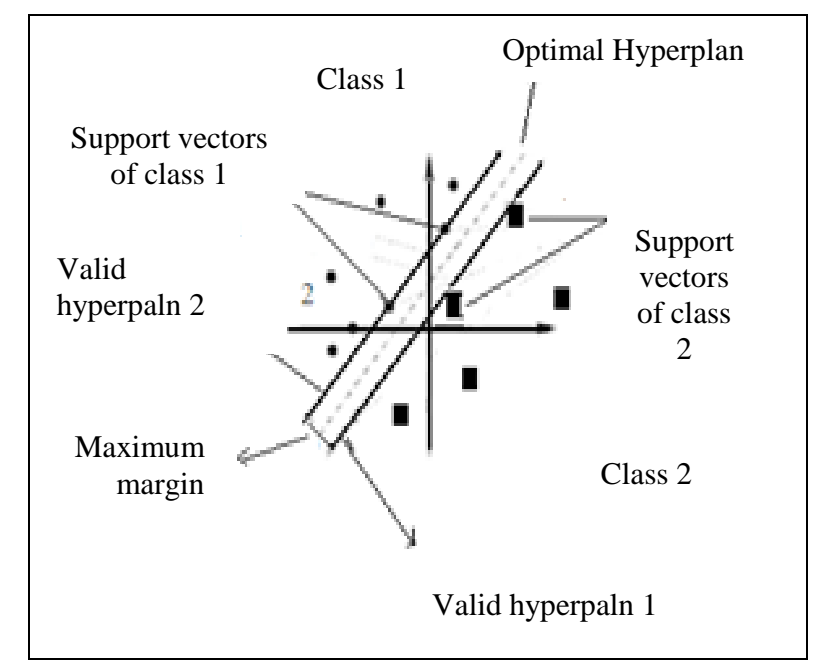

\section{Figure 4. Linear Support Vectors Machines Illustration}

In fact, the nearest vectors that only is used for finding this classifier and is called the support vectors. However the property of SVM is that this classifier must be optimal, in other words it must maximizes the distance between support vectors of a class and those of other. The classifier is represented by a special function named a decision function:

$$
\begin{gathered}
f: \mathfrak{R}^{n} \times \mathfrak{R}^{n} \times \mathfrak{R} \rightarrow \mathfrak{R} \\
x, w, b \rightarrow \mathfrak{R}
\end{gathered}
$$

Where $x \in R^{n}, y \in\{-1,1\} w$ and $b$ are the parameters of the classifier and $y$ is the label.

For maximizing the distance between the supports vectors of a class and those of other class, it must to solve a minimization problem under constraints called the primal problem:

$$
\begin{array}{ll}
\text { To minimize } & \frac{1}{2}\|w\|^{2} \\
\text { Subject to } & y_{i}\left(w x_{i}+b\right) \geq 1, \quad \forall i=1,2, \ldots . ., n
\end{array}
$$

The Lagrangian operator of the primal problem is:

$$
L(w, b, \alpha)=\frac{1}{2}\|w\|^{2}-\sum_{i=1}^{n} \alpha_{i}\left[y_{i}\left(w x_{i}+b\right)-1\right]
$$

The dual variables $\alpha_{i}$ are called Lagrange multipliers. The associated dual problem is: 
To maximize $D(\alpha)=\sum_{i=1}^{n} \alpha_{i}-\frac{1}{2} \sum_{i, j} \alpha_{i} \alpha_{j} y_{i} y_{j} K\left(x_{i}, x_{j}\right)$

$$
\text { Subject to } \sum_{i=1}^{n} \alpha_{i} y_{i}=0,0 \leq \alpha_{i} \leq C \quad \forall i=1,2, \ldots ., n
$$

Only the $\alpha_{i}{ }^{*}$ corresponding to the support vectors is nonzero, the decision function associated to this separation is:

$$
f(x)=\sum_{i=1}^{n} \alpha_{i}^{*} y_{i} K\left(x, x_{i}\right)+b
$$

Where $\mathrm{K}$ is called kernel function, some examples are :

$$
\begin{array}{ll}
\text { Kernel linear } & (a x y+b)^{n} \\
\text { Kernel polynomial of degree } \mathrm{n} & e^{-\frac{\|x-y\|^{2}}{2 \sigma^{2}}}
\end{array}
$$

The method presented above is used only for a binary problem; in fact several studies have generalized of the SVM to N classes.Among them, we have used in this approach a one that is called one against all based to use $\mathrm{N}$ ( $\mathrm{N}=28$ number of Arabic characters) decision functions allowing to make a discrimination of a class labeled by a value equal to 1 and containing a one vector against all other vectors grouped in a other class opposite having a label -1 . In the classification phase, the value image of an unknown vector $\mathrm{X}$ (test character) is calculated by all $\mathrm{N}$ decision functions which are already saved in learning phase. The recognition will be attributed to character that the decision function separates its class to another class that contains the rest of characters which gives the highest value.

$$
\text { Class }(X)=\arg \underset{i=1,2 \ldots N}{\max (\underset{i}{f}(X))}
$$

\section{Experiments and Results}

Firstly, we present an image of some isolated handwritten Arabic characters. 


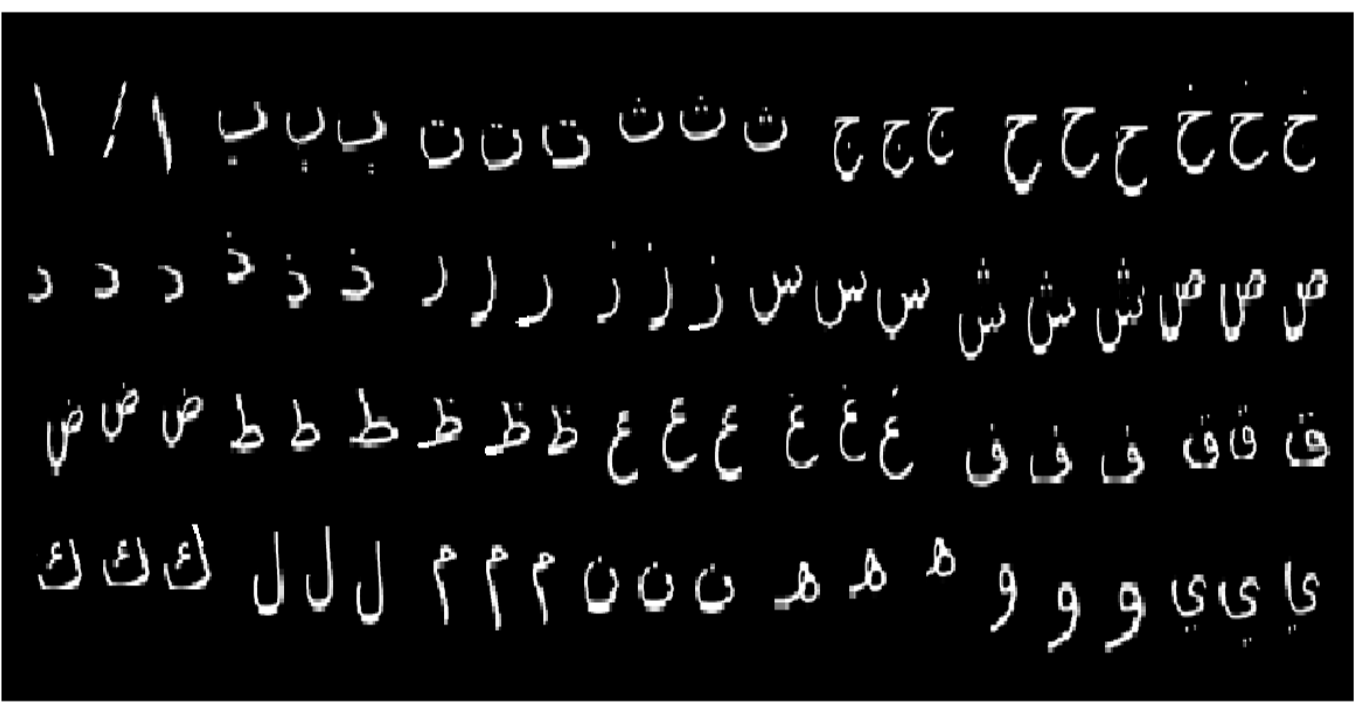

Figure 5. Example of some Isolated Handwritten Arabic Characters

The desired goal is to compare between the performances that is to say the precision (rate recognition) and speediness or rapidity (time of execution) of six hybrid methods of features extraction cited prouvesiouly.

In order to achieve the desired comparison, we have used the following data's:

- Each numeral image has a size equal to 30x30 pixels.

- The number of images of learning base is equal to 500 while that of test base is equal to 2500 .

- The Racah parametrs are equal to $a=2, b=34, \alpha=4, \beta=5$

- The Gegenbauer parameter is equal to $\lambda=0.5$.

- The Hahn parametrs are equal to $\mu=1, v=2$.

- The kernel function chosen is the GRBF with a standard deviation equal to $\boldsymbol{\sigma}=0.5$.

- each character is converted to a obtained by the following relationship:

$$
V_{c}=V_{\text {profile }} \bigcup V_{\text {moment }}
$$

Therefore, we summarized the values that we obtained of the recognition rate $\tau_{c}$ of each character (given in \%) and of the global rate $\tau_{g}$ of all characters (given in \%) and the global time of execution $t_{g}$ (given in $\%$ ) in the following table knowing that :

- $\mathrm{P}+\mathrm{R}$ means to profile method combined with Racah moment.

- $\mathrm{P}+\mathrm{G}$ means to profile method combined with Gegenbauer moment.

- $\mathrm{P}+\mathrm{H}$ means to profile method combined with Hahn moment.

- $\mathrm{P}+\mathrm{T}$ means to profile method combined with Tchebychev moment.

- P+OFM means to profile method combined with Orthogonal Fourier-Mellin moment.

- $\mathrm{P}+\mathrm{R}+\mathrm{G}+\mathrm{H}+\mathrm{T}+\mathrm{OFM}$ means to profile method combined in same time with Racah, Gegenbauer, Hahn, Tchebychev and Orthogonal Fourier-Mellin moments. 
Table 1. The Obtained Recognition Rates for each Hybrid Method

\begin{tabular}{|c|c|c|c|c|c|c|}
\hline Characters & $\begin{array}{c}\tau_{c} \\
(P+R)\end{array}$ & $\begin{array}{c}\tau_{c} \\
(P+G)\end{array}$ & $\begin{array}{c}\tau_{c} \\
(P+H)\end{array}$ & $\begin{array}{c}\tau_{c} \\
(P+T)\end{array}$ & $\tau_{c}(P+O F M)$ & $\begin{array}{c}\tau_{c} \\
(P+R+G+H+T+O F M)\end{array}$ \\
\hline & 92.34 & 90.15 & 88.58 & 81.25 & 78.82 & 95.00 \\
\hline & 89.67 & 86.55 & 82.00 & 80.34 & 79.55 & 93.25 \\
\hline & 81.25 & 80.34 & 75.25 & 78.25 & 75.25 & 90.85 \\
\hline & 90.12 & 85.67 & 80.45 & 81.67 & 77.34 & 91.00 \\
\hline & 81.25 & 75.88 & 77.55 & 74.34 & 70.15 & 82.25 \\
\hline & 78.87 & 81.25 & 80.47 & 77.85 & 78.67 & 85.55 \\
\hline & 80.34 & 80.36 & 76.25 & 75.00 & 80.15 & 86.34 \\
\hline 3 & 91.77 & 85.47 & 83.00 & 82.35 & 79.80 & 93.25 \\
\hline & 92.67 & 90.55 & 87.77 & 87.34 & 85.25 & 94.67 \\
\hline & 93.15 & 90.00 & 90.25 & 88.00 & 81.77 & 95.00 \\
\hline & 90.47 & 90.87 & 86.75 & 85.45 & 82.34 & 92.25 \\
\hline & 75.75 & 75.88 & 73.55 & 72.85 & 70.25 & 80.75 \\
\hline & 78.85 & 77.35 & 75.40 & 78.34 & 76.80 & 83.34 \\
\hline & 76.34 & 74.00 & 77.25 & 80.25 & 75.00 & 82.85 \\
\hline 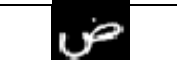 & 84.88 & 80.15 & 81.00 & 77.25 & 73.55 & 87.00 \\
\hline & 78.15 & 76.50 & 75.00 & 72.34 & 70.34 & 85.25 \\
\hline ظط & 79.67 & 77.30 & 76.67 & 75.85 & 74.80 & 86.00 \\
\hline$\varepsilon$ & 78.25 & 75.70 & 77.55 & 75.67 & 73.55 & 84.75 \\
\hline$\varepsilon$ & 74.47 & 70.85 & 70.00 & 70.55 & 71.67 & 84.25 \\
\hline & 82.85 & 81.77 & 82.15 & 80.55 & 83.80 & 85.75 \\
\hline c9 & 90.55 & 86.88 & 85.95 & 84.25 & 80.50 & 93.67 \\
\hline$\Leftrightarrow$ & 91.34 & 90.34 & 85.10 & 85.00 & 80.85 & 92.25 \\
\hline J & 92.77 & 88.75 & 86.15 & 85.15 & 82.34 & 93.80 \\
\hline 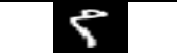 & 93.10 & 90.25 & 86.55 & 85.25 & 81.00 & 94.00 \\
\hline$\vec{c}$ & 89.25 & 87.55 & 85.25 & 84.00 & 80.55 & 91.25 \\
\hline$\infty$ & 88.12 & 85.00 & 84.17 & 82.45 & 78.15 & 90.34 \\
\hline 9 & 83.47 & 78.00 & 76.20 & 75.85 & 76.55 & 85.00 \\
\hline 5 & 89.34 & 86.25 & 84.34 & 83.45 & 80.45 & 92.67 \\
\hline$\tau_{g}$ & 85.32 & 82.84 & 81.09 & 80.03 & 77.83 & 89.01 \\
\hline$u_{g}$ & 786.55 & 652.73 & 734.57 & 674.18 & 560.71 & 3118.7 \\
\hline
\end{tabular}


The graphical representation associated to recognition rate of each character for each hybrid method is:

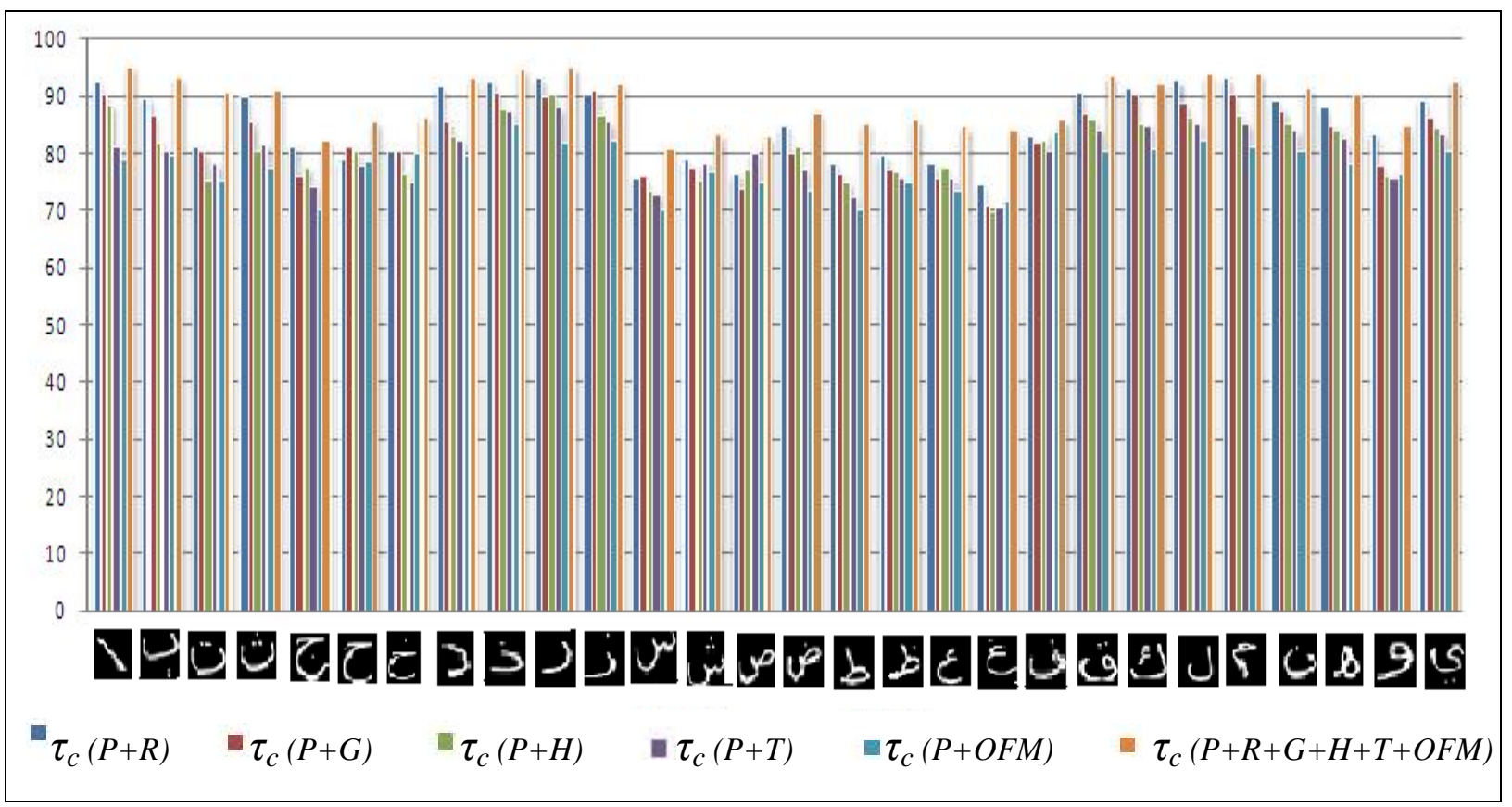

Figure 6. The Graphical Representation of Recognition Rate of each Character for each Hybrid Method

Then the graphical representation associated to global rate recognition is presented as follow:

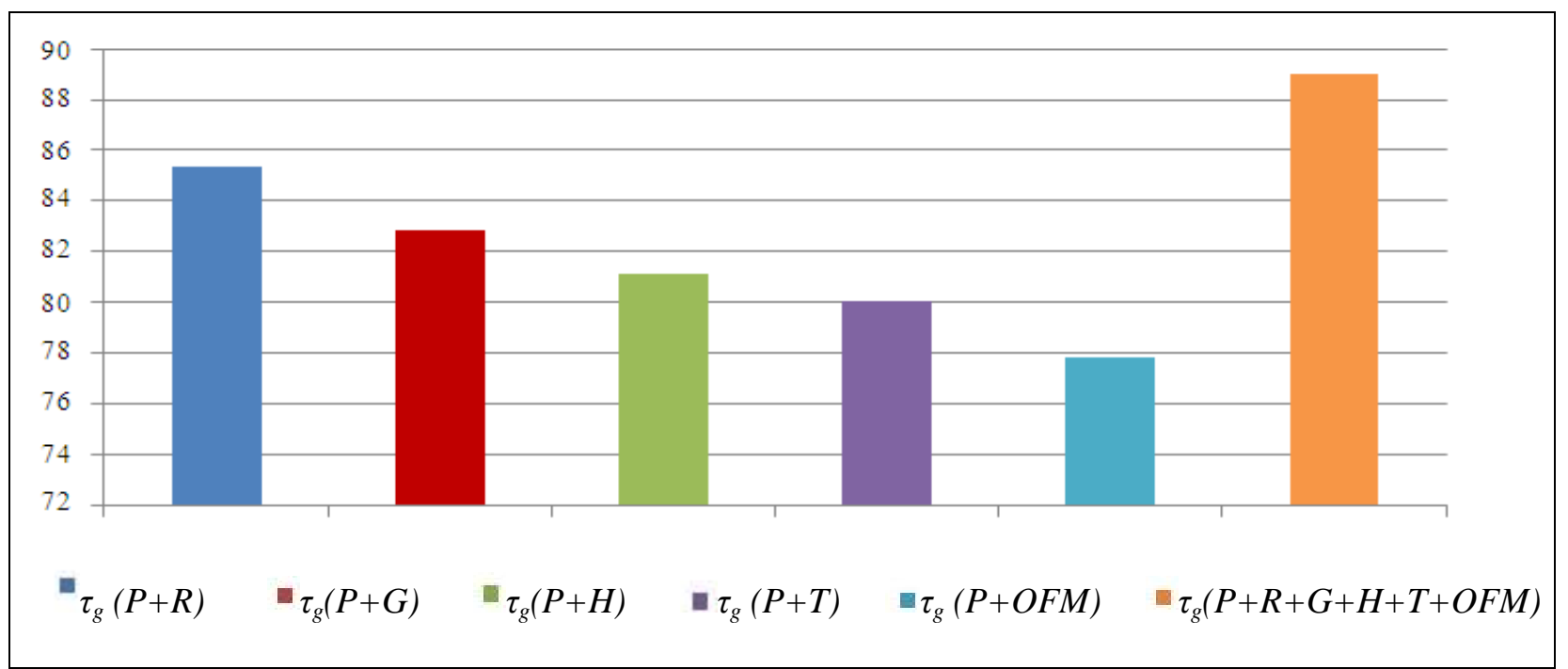

Figure 7. The Graphical Representation of Global Rate Recognition for each Hybrid Method

Moreover the associated graphical representation to time of execution is presented as follow: 


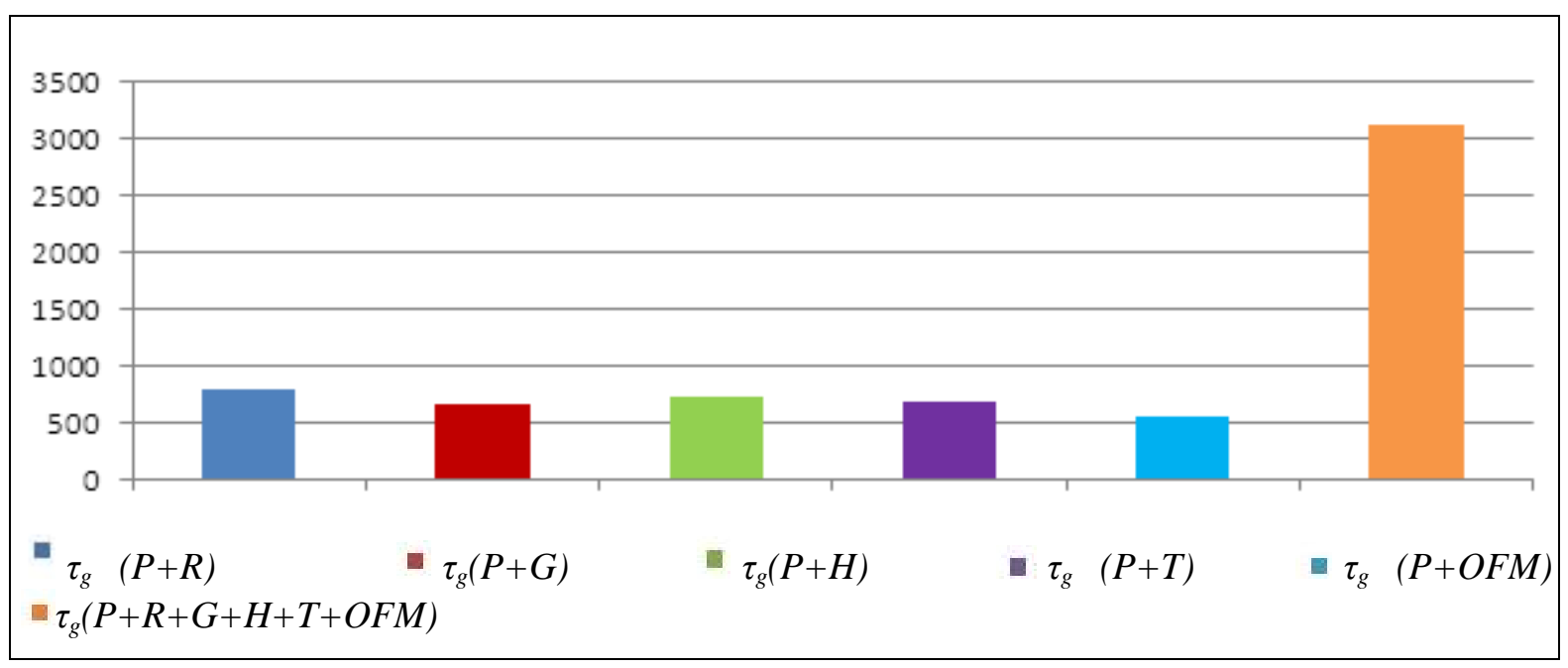

Figure 8. The Global Time of Execution for each Hybrid Method

\section{$\diamond \quad$ Interpretation:}

Taking into account all the results that have we obtained, we concluded that:

- The most precise hybrid method the profile method combined in the same time with Racah, Gegenbauer, Hahn, Tchebychev and orthogonal Fourier-Mellin moments followed by profile with Racah moment then profile with Gegenbauer moment then profile with Hahn moment then profile with Tchebychev moment followed finally by profile with orthogonal Fourier-Mellin moment.

- The most fast hybrid method the profile method combined orthogonal Fourier-Mellin moment followed by profile with Gegenbauer moment then profile with Tchebychev moment then profile with Hahn moment then profile with Racah moment followed finally by profile combined with all these moments

For this purpose, more precisely to fix the ideas, we note:

The difference of precision between both hybrid methods $h m_{l}$ and $h m_{2}$ is:

Therefore:

$$
\Delta P=\tau_{g_{, h m_{1}}}-\tau_{g, h m_{2}}
$$

- If $\Delta P>0$ we will have a gain of precision, in this case $\Delta P$ is called the rate of growth of precision.

- If $\Delta P<0$ we will have a loss of precision, in this case $\Delta P$ is called the rate of decay of precision. $h m_{2}$ by:

In a like manner, we note the difference of speediness (rapidity) between $h m_{l}$ and

$$
\Delta S=t_{g_{, h m_{1}}}-t_{g_{, h m_{2}}}
$$

- If $\Delta S>0$ we will have a advancement of speediness or of rapidity. in this case $\Delta S$ is called the rate of decay of speediness.

- If $\Delta \mathrm{S}<0$ we will have a delay of rapidity. in this case $\Delta S$ is called the rate of growth of speediness. 
Hence, the following table presents different values of $\triangle P$ (gain of precision) and $\Delta F$ (loss of speediness):

Table 2. The Different Obtained Values of Gain of Precision and Loss of Speediness

\begin{tabular}{|c|c|c|}
\hline $\mathbf{h m}_{\mathbf{1}}, \mathbf{c l}_{\mathbf{1}} ; \mathbf{h m}_{\mathbf{2}}, \mathbf{c l}_{\mathbf{2}}$ & $\Delta P(\boldsymbol{\%})$ & $\Delta F$ (second) \\
\hline $\mathrm{P}+\mathrm{R}+\mathrm{G}+\mathrm{H}+\mathrm{T}+\mathrm{OFM} ; \mathrm{P}+\mathrm{R}$ & 03.69 & 2332 \\
\hline $\mathrm{P}+\mathrm{R}+\mathrm{G}+\mathrm{H}+\mathrm{T}+\mathrm{OFM} ; \mathrm{P}+\mathrm{G}$ & 06.17 & 2466 \\
\hline $\mathrm{P}+\mathrm{R}+\mathrm{G}+\mathrm{H}+\mathrm{T}+\mathrm{OFM} ; \mathrm{P}+\mathrm{H}$ & 07.92 & 2384 \\
\hline $\mathrm{P}+\mathrm{R}+\mathrm{G}+\mathrm{H}+\mathrm{T}+\mathrm{OFM} ; \mathrm{P}+\mathrm{T}$ & 08.98 & 2444 \\
\hline $\begin{array}{c}\mathrm{P}+\mathrm{R}+\mathrm{G}+\mathrm{H}+\mathrm{T}+\mathrm{OFM} ; \\
\mathrm{P}+\mathrm{OFM}\end{array}$ & 11.18 & 2558 \\
\hline
\end{tabular}

Therefore the table above shows that certainly to obtain a certain gain in precision means to a loss in speediness (delay in time of execution).

\section{Conclusion}

The obtained results in the recognition of isolated handwritten Arabic characters show that reliable recognition is possible by using the median filter, the thresholding, the centering and the edge detection techniques in pre-processing phase, the profile method combined respectively with Racah, Gegenbauer, Hahn, Tcebychev and orthogonal Fourier-Mellin moments then with all these descriptors in the same time in features extraction phase and the SVM in the recognition phase. Our goal in this study is to compare between the performances in terms of precision and speediness of all these hybrid methods. Considering that the results that we have obtained, we concluded that the most precise but the slowest hybrid method is profile combined with all these five moments.

\section{Acknowledgements}

In conclusion we are very grateful to our professors Mister Said Safi and Mister Belaid Bouikhalene for their encouragements, their cooperation, their advices, their guidance in the realization of this work. Many thanks again to them.

\section{References}

[1] H. Zhu and H. Shu, "Image analysis by discrete orthogonal Racah moments", Signal Processing, vol. 87, (2007), pp. 687-708.

[2] G. Szego, "Orthogonal polynomials", American Mathematical Society Providence, Rhode Island, (2000).

[3] K. M. Hosny, "Image representation using accurate orthogonal Gegenbauer moments", Pattern Recognition Letters, vol. 32, no. 6, (2011) April 15, pp.795-804.

[4] J. Zhou and H. Shu, "Image analysis by discrete orthogonal Hahn moments", M. S. Kamel, A. C. Campilho, (eds.) ICIAR 2005. LNCS, Springer, Heidelberg, vol. 3656, (2005), pp. 524-531.

[5] R. Mukundan, "Image analysis by Tchebichef moments", IEEE Image Analysis by Tchebichef Moments, vol. 10, no. 9, (2001), pp. 1357-1364.

[6] Y. Sheng and L. Shen, "Orthogonal Fourier-Mellin moments for invariant pattern recognition", Journal of the Optical Society of America A, vol. 11, no. 6, (2007), pp. 1748-1757. 
[7] B. V. Dhandra, R. G. Benne, M. Hangarge and B. Kannada, "Telugu and Devanagari Handwritten Numeral Recognition with Probabilistic Neural Network: A Novel Approach”, IJCA Special Issue on "Recent Trends in Image Processing and Pattern Recognition", RTIPPR, (2010).

[8] R. Kumar and K. Kumar Ravulakollu, "On the Performance of Devnagari Handwritten Character Recognition”, World Applied Sciences Journal, 2014, ISSN 1818-4952, () IDOSI Publications (2014), vol. 31, no. 6, (2014), pp. 1012-1019.

[9] M. Hosalli Ramappa1 and S. Krishnamurthy, "A Comparative Study of Different Feature Extraction and Classification Methods for Recognition of Handwritten Kannada Numerals", International Journal of Database Theory and Application, vol. 6, no. 4, (2013) August.

[10] M. A. Abu-Zanona and B. M. El-Zaghmouri, "Current Arabic (Hindi) Hand Written Numbers Segmentaion and Recognition Advance Image Processing and Neural Network", Journal of Emerging Trends in Computing and Information Sciences, vol. 3, no. 6, (2012) June.

[11] R. Salouan, S. Safi and B. Bouikhalene, "A Comparative Study between the Pseudo Zernike and Krawtchouk Invariants Moments for Printed Arabic Characters Recognition”, Journal of Emerging Technologies in Web Intelligence, vol. 6, no. 1, (2014) February.

[12] R. Salouan, S. Safi and B. Bouikhalene, "Printed Arabic Noisy Characters Recognition Using the Multi-layer Perceptron", International Journal of Innovation and Scientific Research ISSN 2351-8014, (2014) Innovative Space of Scientific Research Journals, vol. 9, no. 1, (2014) September, pp. 61-69.

[13] H. Zhu and H. Shu, "Image analysis by discrete orthogonal Racah moments", Signal Processing, vol. 87, (2007), pp. 687-708.

[14] T. Xia and H. Zhu, "Image description with generalized pseudo-Zernike moments", Journal of the Optical Society of America, vol. 24, no. 1, (2007), pp. 50-59.

[15] R. Salouan, S. Safi and B. Bouikhalene, "A Comparison between the Self-Organizing Maps and the Support Vector Machines for Handwritten Latin Numerals Recognition", International Journal of Innovation and Scientific Research ISSN 2351-8014, (C) 2014 Innovative Space of Scientific Research Journals, vol. 7, no. 1, (2014) August, pp. 50-56.

[16] R. Salouan, S. Safi and B. Bouikhalene, "A Comparative Study Between the Hidden Markov Models and the Support Vector Machines for Noisy Printed Numerals Latin Recognition", International Journa of Innovation and Scientific Research ISSN 2351-8014, () (2014) Innovative Space of Scientific Research Journals, vol. 5, no. 1, (2014) July, pp. 16-24.

[17] R. Salouan, S. Safi and B. Bouikhalene, "Printed Eastern Arabic Noisy Numerals Recognition Using Hidden Markov Model and Support Vectors Machine", International Journal of Innovation and Applied Studies ISSN 2028-9324, ( ) (2014) Innovative Space of Scientific Research Journals, vol. 9, no. 3, (2014) November, pp. 1032-1042.

[18] R. Salouan, S. Safi and B. Bouikhalene, "Isolated Handwritten Roman Numerals Recognition using Dynamic Programming, Naïve Bayes and Support Vectors Machines", International Journal of Computer Applications (0975 - 8887), vol. 113, no. 19, (2015) March.

[19] V. N. Vapnik, "An overview of statistical learning theory", IEEE Trans. Neural Networks, vol. 10, (1999) September, pp. 988-999. 
International Journal of Advanced Science and Technology Vol.78 (2015) 